\title{
"X-8": An Experimental Interactive Toy to Support Turn-Taking Games in Children with Autism Spectrum Disorders
}

\author{
Beste Özcan ${ }^{(凶)}(\mathbb{D}$, Valerio Sperati®, Flora Giocondo®, \\ and Gianluca Baldassarre \\ Institute of Cognitive Sciences and Technologies National Research Council \\ of Italy ISTC-CNR, Rome, Italy \\ \{beste.ozcan, valerio.sperati,flora.giocondo, \\ gianluca.baldassarre\}@istc.cnr.it
}

\begin{abstract}
Turn-taking is a type of interaction where two individuals alternate a behaviour (e.g. during a conversation). Such competence which is a foundamental key in the social behaviour - is often compromised in children with Autism Spectrum Disorders (ASDs). Neurodevelopmental therapists report how it is extremely difficult to teach this skill to young ASDs, yet it would be very important. In the current proposal we present an early prototype of interactive toy called $X-8$ : it is a soft, wearable, six-legged octopus able to distinguish between two people, and produce different luminous patterns according to the user who is touching it. The toy can then potentially support therapists in set up sensory-motor games based on turn-taking rules. A first proof of concept is described and possible activities are proposed.
\end{abstract}

Keywords: Autism spectrum disorders $\cdot$ Turn-taking $\cdot$ Interactive technology $\cdot$ Transitional wearable companion $\cdot$ Therapy

\section{Introduction}

Autism Spectrum Disorder (ASD) is a neurodevelopmental condition characterised by important life-long impairments in the social, communicative and affective areas [1]. Early rehabilitative interventions - possibly within the first years of life - have proved to be effective in ameliorating the severity of the condition. Probably due to the plasticity on the nervous system [5], behavioural treatments are in fact more effective at a young age, in stimulating and strenghtening the basic social competences which are critical for the cognitive development (e.g. imitation, eye-contact, joint attention [7]).

In this context, interactive technologies seem to be a promising tool to support the therapeutic activity [2]. In fact, it was observed that most of children diagnosed with autism (from now on ASDs), show a clear attraction toward

(C) The Author(s) 2021

C. Stephanidis et al. (Eds.): HCII 2021, CCIS 1419, pp. 233-239, 2021.

https://doi.org/10.1007/978-3-030-78635-9_32 
technological devices. This intrinsic interest can then be exploited to maintain an high child's engagement during the therapeutic activities.

In this work-in-progress we present a simple - yet interesting - experimental, interactive toy called $X-8$. The prototype is designed to support turn-taking games, namely playful activities where two people (the child and the therapist in our case) must take turns. Turn-taking is a general social competence, often impaired in ASDs, which is pivotal for all communications activities featuring a reciprocal back-and-forth exchange [4]; this skill relies in facts on a complex social-cooperative coupling, precise in time, and based on several signals (visual, auditory, verbal and nonverbal) [8].

Surely the proposed device does not want to compete with much more sophisticated tools (as humanoid robots); yet we argue it can be still interesting for therapists, because of its simplicity, ease of use, and versatility as a potential supporting tool.

\section{Related Works}

Innovative technologies (e.g. interactive environments, robotic platforms, virtual reality) seem to be effective to support the therapeutic activity in ASD. [2]. About the turn-taking, robots have been used in some promising, exploring studies $[6,13]$. Unexpectedly, very few works were found, which exploit simpler - yet interesting enough - technological devices other than robots, to train the turn-taking competence in ASDs.

In [9] the authors developed an interactive system called COLOLO composed of two wirelessly connected spherical toys; the spheres (one held by the therapist and one by the child) are designed to emit luminous and vibrational feedback if the turn-taking game roles are complied. A similar approach, based on interactive lighting blocks which interact when positioned in each other's vicinity, was used in $[3]$.

In the current work-in-progress we focused on a single device; in particular, we wanted to explore the technical possibility for the proposed toy, to detect autonomously the identity of the two players, namely the child and the therapist, so to respond differently according to the role in the game. We then present a simple proof of concept, showing the potential of the device in supporting turntaking activities.

\section{Design Concept of "X-8" First Prototype}

\subsection{Design Considerations}

The design of $X-8$ is based on the concept of Transitional Wearable Companion (TWC) [10]. A TWC is an interactive "smart" toy with the following features: (i) it is potentially able to arouse emotional attachment and reassuring feelings, thanks to its soft material and the wearability characteristic; (ii) it can be perceived as a playmate, thanks to the pleasant animal shape; (iii) it can stimulate simple social games (based on imitation and joint attention), exploiting the 

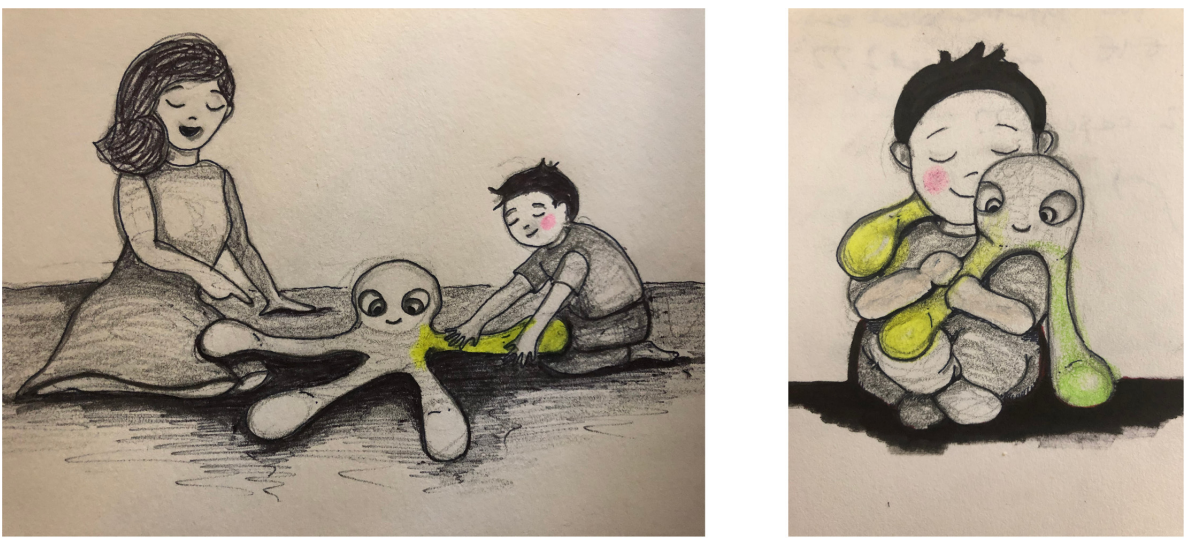

Fig. 1. On left, the turn-taking concept: $X-8$ can emit different lights and sounds according to the user who touches its tentacles. On right, the wearability concept: the tentacles, thanks to small weights, adapt to shoulders and gently hug the child, so that $X-8$ can be easily worn around the neck.

attractiveness of coloured lights and sounds, supported by the inner electronics (see Fig. 1). The concept of TWC - as a potential supporting tool in therapy is currently in a promising experimentation phase on young children diagnosed with ASD [11].

$X-8$ was designed as a six-legged octopus (which makes it a very special octopus!). Textile materials (external cotton envelope, internal soft padding) are hypoallergenic and fireproof. Within each leg there is a small pocket filled with linen seeds: these pouches make legs a little bit heavier, so that $X-8$ can easily adapt its shape if placed on human body, and can consequently be comfortably carried on the child's shoulders. About this point, it was interestingly observed how weighted vests can exert a calming effect on some ASD children [12]. Pockets with linen seeds have another additional, appealing feature: they could be taken out, heated in microwave, and placed back, so that $X-8$ becomes pleasantly warm, so enhanching the potential reassuring effects of the toy.

Concerning the interactive behaviour, each tentacle is able to respond to human touch emitting coloured lights (and brief amusing sounds in the next version of the prototype), namely sensory stimuli which are very rewarding for young children. The toy is able to autonomously detect the user's identity: in the context of an hypotetical therapy session including two actors, $X-8$ can "understand" if its tentacles are touched by the child or the therapist. This feature makes it possible to set up several games based on turn-taking rules.

\subsection{Technical Specifications}

In this first prototype, $X-8$ electronics is based on a Arduino Uno board that (once engineered and miniaturised) will be hidden in the octopus body. Luminous 
patterns are emitted through embedded addressable leds, nicely diffused by the soft padding. The device can detect the human touch through capacitive sensors and conductive patches, stiched on the six tentacles. In order to discriminate between the users touches, each tentacle presents also a magnetic sensor: if the therapist wears a ring embedding a little magnet, his/her touch will activate both the capacitive and magnetic sensors (see Fig. 2). This simple system lets $X-8$ to autonomously "understand" who is touching its tentacles. The solution obviously works only for two users, but we think it is not an important limitation, as many turn-taking activities are based on two people only. A first proof-ofconcept, showing the feasibility of the solution, can be observed at this link https://bit.ly/31lfUv3.
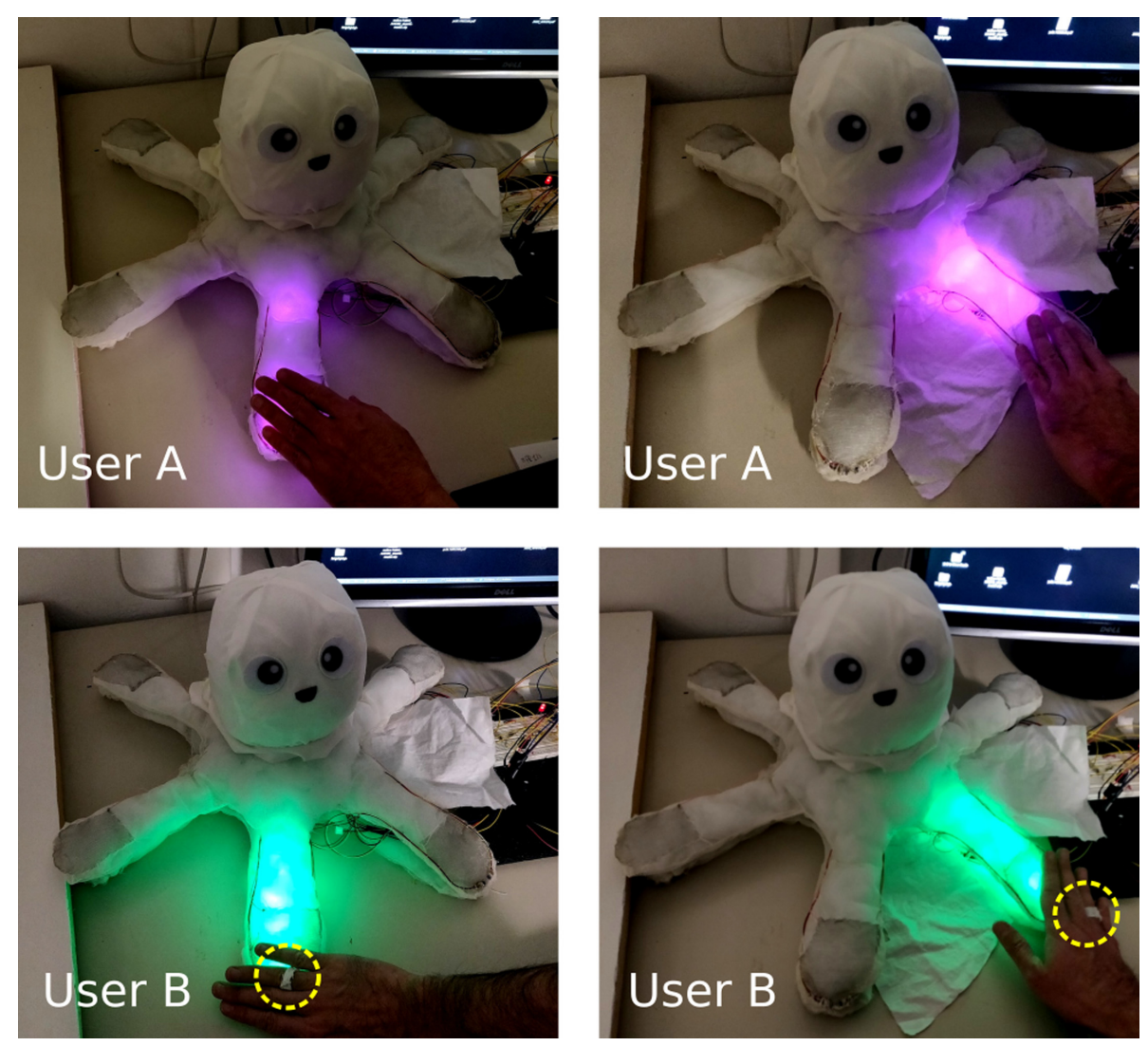

Fig. 2. In this functional test, the toy responds with magenta color to user $A$ and with green to user $B$. The discernment is possible thanks to the small, discreet magnetic ring worn by user $B$ (highlighted in yellow) which is detected by the sensors. The shown prototype, as a work-in-progress, is still partially disassembled: all electronics will be hidden by an external envelope. (Color figure online) 


\subsection{Proposals on Possible Games}

Once the device is able to detect the identity of the users, several games based on turn-taking rules can be conceived, i.e. they can be easily implemented in the device software. As an example, if we figure a typical therapeutic session, with child and therapist sitting next to each other, with $X-8$ placed in the middle (floortime therapy), the following proposals, featuring an increasing complexity, can be imagined:

- Proposal 1: different colors are associated to each user, e.g. magenta to the child and green to the therapist (as in Fig. 2). The child can touch the toy and make it glow in magenta, but he/her has to wait for therapist's touch to observe the toy glowing in green (hopefully, a desired outcome which the child wants to observe too). This is obviously the most simple activity, and it is already possible with the current prototype.

- Proposal 2: the toy glows briefly in magenta, indicating that it is the child's turn to touch the toy. If he/she touches any of the tentacles, $X-8$ emits a generalised rewarding pattern, featuring several colours on all legs (as a rainbow); then the toy turns dark and, after few seconds, glows briefly in green, indicating that now it is the therapist's turn. If the child touches the toy in the place of the therapist, $X-8$ recognises the violation of the rule, and does not produce any reward pattern. This activity is more complicated, and features a fixed sequence of turns: first the child, then the therapist, then all over again.

- Proposal 3: a random tentacle glows intermettently in green or magenta, indicating which user has to touch the active tentacle. If the rule is respected (i.e. the correct user touches the correct leg) $X-8$ emits a generalised luminous rewarding pattern; then the game starts again with another random tentacle and a random colour (green or magenta). This activity is more complex than previous one, as it requires more attentional resources and features a non-fixed sequence of turns.

The above mentioned games are only proposals, made possible by improving the $X-8$ software. It is easily feasible then further improve the system with the addition of a tablet, connected to the toy via Bluetooth, where the therapist can select - through an App - which game is more adapt to the child.

\section{Conclusion and Future Work}

We presented the first version of a toy prototype, which can potentially support turn-taking activities in young children diagnosed with ASD. In the next phase we are going to complete the device, through the following steps: (I) the improvement of the inner electronics - also with the addition of auditory feedback - to make the toy usable in a real therapeutic session; (II) the design of several, different games, through the development of a specific software; (III) the 
development of a control App, to let the therapist to select the current game, and save the behavioural data for research purpose.

Once the toy is competed, we plan to test the effectiveness of $X-8$ in a pilot experimental session involving a therapist and a child diagnosed with ASD. This phase will be carried out in collaboration with neurodevelopmental researchers. Our main goal is to provide the therapist with an effective, versatile, easy-to-use tool, which can be helpful in training and reinforcing the turn-taking competences in this type of children.

Acknowledgments. This work has received funding from the European Union's Horizon 2020 Research and Innovation program under grant agreements No. 94587 (project PlusMe: Transitional Wearable Companions for the therapy of children with Autism Spectrum Disorders) and No. 952095 (project IM-TWIN: from Intrinsic Motivations to Transitional Wearable INtelligent companions for autism spectrum disorder).

\section{References}

1. American Psychiatric Association: Diagnostic and Statistical Manual of Mental Disorders: DSM-5. Washington DC, 5th edn. (2013). https://doi.org/10.1176/appi. books.9780890425596

2. Boucenna, S., et al.: Interactive technologies for autistic children: a review. Cogn. Comput. 6(4), 722-740 (2014). https://doi.org/10.1007/s12559-014-9276-x

3. Brok, J.C.J., Barakova, E.I.: Engaging autistic children in imitation and turntaking games with multiagent system of interactive lighting blocks. In: Yang, H.S., Malaka, R., Hoshino, J., Han, J.H. (eds.) ICEC 2010. LNCS, vol. 6243, pp. 115-126. Springer, Heidelberg (2010). https://doi.org/10.1007/978-3-642-15399-0_11

4. Holler, J., Kendrick, K.H., Casillas, M., Levinson, S.C.: Editorial: turn-taking in human communicative interaction. Front. Psychol. 6(DEC), 1-4 (2015). https:// doi.org/10.3389/fpsyg.2015.01919

5. Izadi-Najafabadi, S., Rinat, S., Zwicker, J.G.: Rehabilitation-induced brain changes detected through magnetic resonance imaging in children with neurodevelopmental disorders: a systematic review. Int. J. Dev. Neurosci. 73(November 2018), 66-82 (2019). https://doi.org/10.1016/j.ijdevneu.2018.12.001

6. Kozima, H., Nakagawa, C.: Social robots for children: practice in communicationcare. In: International Workshop on Advanced Motion Control, AMC 2006, pp. 768-773 (2006). https://doi.org/10.1109/AMC.2006.1631756

7. Neimy, H., Pelaez, M., Carrow, J., Monlux, K., Tarbox, J.: Infants at risk of autism and developmental disorders: establishing early social skills. Behav. Dev. Bull. 22(1), 6-22 (2017). https://doi.org/10.1037/bdb0000046

8. Noel, J.P., De Niear, M.A., Lazzara, N.S., Wallace, M.T.: Uncoupling between multisensory temporal function and nonverbal turn-taking in autism spectrum disorder. IEEE Trans. Cogn. Dev. Syst. 10(4), 973-982 (2018). https://doi.org/10. 1109/TCDS.2017.2778141

9. Nunez, E., Matsuda, S., Hirokawa, M., Yamamoto, J., Suzuki, K.: Effect of sensory feedback on turn-taking using paired devices for children with ASD. Multimodal Technol. Interact. 2(4), 1-18 (2018). https://doi.org/10.3390/mti2040061 
10. Özcan, B., Caligiore, D., Sperati, V., Moretta, T., Baldassarre, G.: Transitional wearable companions: a novel concept of soft interactive social robots to improve social skills in children with autism spectrum disorder. Int. J. Soc. Robot. 8(4), 471-481 (2016). https://doi.org/10.1007/s12369-016-0373-8

11. Sperati, V., et al.: Acceptability of the transitional wearable companion "+me" in children with autism spectrum disorder: a comparative pilot study. Front. Psychol. 11(May), 1-9 (2020). https://doi.org/10.3389/fpsyg.2020.00951

12. Stephenson, J., Carter, M.: The use of weighted vests with children with autism spectrum disorders and other disabilities. J. Autism Dev. Disord. 39(1), 105-114 (2009). https://doi.org/10.1007/s10803-008-0605-3

13. Wainer, J., Robins, B., Amirabdollahian, F., Dautenhahn, K.: Using the humanoid robot KASPAR to autonomously play triadic games and facilitate collaborative play among children with autism. IEEE Trans. Auton. Ment. Dev. 6(3), 183-199 (2014). https://doi.org/10.1109/TAMD.2014.2303116

Open Access This chapter is licensed under the terms of the Creative Commons Attribution 4.0 International License (http://creativecommons.org/licenses/by/4.0/), which permits use, sharing, adaptation, distribution and reproduction in any medium or format, as long as you give appropriate credit to the original author(s) and the source, provide a link to the Creative Commons licence and indicate if changes were made.

The images or other third party material in this chapter are included in the chapter's Creative Commons licence, unless indicated otherwise in a credit line to the material. If material is not included in the chapter's Creative Commons licence and your intended use is not permitted by statutory regulation or exceeds the permitted use, you will need to obtain permission directly from the copyright holder.

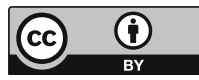

